\title{
LA CARACTERIZACIÓN ECONÓMICA DE LA LENGUA Y SU RELACIÓN CON EL CAPITAL SOCIAL
}

\section{THE ECONOMIC CHARACTERIZATION OF LANGUAGE AND ITS RELATION WITH SOCIAL CAPITAL}

José Manuel Maneiro

Universidad de Alcalá de Henares. España

josem.maneiro@uah.es

Daniel Sotelsek

Universidad de Alcalá de Henares. España

daniel.sotelsek@uah.es

\section{RESUMEN}

En el contexto de su caracterización económica, la lengua puede ser tratada como un bien preferente y por lo tanto se puede considerar como la precondición para la existencia del capital social. Por otra parte, ese capital social provoca que la pertenencia a un grupo tenga efectos beneficiosos para sus miembros, lo cual sucede en casos de agregados humanos diversos, como una empresa, un barrio, una ciudad. Estos efectos beneficiosos se traducen en mejoras de la eficiencia productiva en el caso de empresas y mejoras en el estatus socioeconómico de los individuos. El lenguaje, por tanto, tiene un papel importante en la creación de capital social, y esto es especialmente importante en el caso de los inmigrantes que residen en un país con una lengua distinta a la suya.

\section{Palabras Clave Adicionales}

Bienes preferentes, Capital Social, Economía del lenguaje, Externalidades de red, Fallos de mercado.

\section{Abstract}

In the context of its economic characterization, language can be treated as a merit good, and can, therefore, be considered the precondition for the existence of social capital. Moreover, social capital makes belonging to a certain group beneficial to its members when referring to human aggregates such as a company, a neighbourhood or a city. These beneficial effects lead to improvements in production efficiency in the case of companies and an increase in socioeconomic status in the case of individuals. Thus, language has an important role in the creation of social capital, which is especially important to immigrants in a country where a different language is spoken.

\section{Additional Keywords}

Language Economy, Market Failures, Merit Goods, Network Externalities, Social Capital. 


\section{INTRODUCCIÓN ${ }^{1}$}

La economía del lenguaje ${ }^{2}$ tiene sus antecedentes en una serie de trabajos que utilizan métodos transplantados de la economía. Dos de esas líneas de investigación cobran relevancia para nosotros: en primer lugar, aquellos estudios que abordan la lengua desde un punto de vista étnico y consideran como hipótesis de trabajo que la lengua materna de los individuos los sitúa en distintos grupos, lo cual, a su vez, tiene efectos sobre su estatus socioeconómico. El segundo tema de interés lo constituye la relación entre la lengua y la eficiencia de las empresas.

En este trabajo, nos proponemos analizar estas ideas intentando vincularlas a un concepto que, en los últimos años, ha adquirido especial relevancia en el ámbito de la Economía y de la Sociología, cual es el capital social ${ }^{3}$. En la siguiente sección se realiza una caracterización de la lengua desde el punto de vista económico con el objeto de identificar la naturaleza de un bien complejo. En el tercer apartado se introduce una aproximación al concepto de capital social en un contexto lingüístico. La cuarta sección analiza los efectos del lenguaje sobre el estatus socioeconómico de las personas, interpretando la literatura existente. Finalmente, el apartado quinto trata la relación entre la eficiencia y el lenguaje en el ámbito de la empresa, considerando, a este fin, a la empresa como un grupo humano y al lenguaje como uno de los elementos que forman parte en la creación de capital social. Por último, el artículo se cierra con unas consideraciones finales.

\section{LA CARACTERIZACIÓN ECONÓMICA DE LA LENGUA: UN BIEN COMPLEJO Y PREFERENTE}

Los estudios sobre la Economía del Lenguaje se caracterizan por el empleo de métodos transplantados, en su mayoría, de la Microeconomía moderna y que despiertan interés en nuevas áreas de estudio4. Según Breton (1998), no es posible afirmar que el análisis

\footnotetext{
${ }^{1}$ Daniel Sotelsek agradece al CILAS (Universidad de San Diego, California) por haberlo acogido como Visiting Scholar durante el curso 2008-2009. Sin duda, ello ha contribuido a finalizar este estudio.

${ }^{2}$ Para Grin (1994): "la economía del lenguaje se encuentra dentro del paradigma de la teoría económica y aplica conceptos e instrumentos corrientes de la ciencia económica al estudio de las relaciones entre variables lingüísticas y ... variables económicas". En este trabajo utilizaremos los términos "lengua" $y$ "lenguaje" de una manera indistinta, tomando para ello la definición de la Real Academia de la Lengua Española, para la cual, en una de sus acepciones, ambos vocablos hacen referencia a un "Sistema de comunicación verbal y casi siempre escrito, propio de una comunidad humana".

${ }^{3}$ Según Portes (1998), el poder heurístico del concepto de capital social proviene de dos fuentes: en primer lugar este concepto se centra en las consecuencias positivas de las interacciones dentro de una sociedad, dejando de lado aquellas características menos atractivas de este proceso. En segundo lugar, presenta estas consecuencias positivas dentro del esquema de una discusión mayor sobre el capital en general y llama la atención de cómo formas no-monetarias del mismo pueden ser fuentes importantes de poder e influencia.

${ }^{4}$ Como ejemplos de estas nuevas áreas de interés Breton (1984) menciona Is siguientes: la organización gubernamental, la discriminación racial y de género, las adicciones, el nacionalismo y el lenguaje.
} 
de una cuestión de índole social o política está realizado bajo una óptica económica a menos que se siga la metodología básica de esta disciplina ${ }^{5}$.

En una economía de mercado los recursos se asignan a través de la interacción de los agentes económicos en los mercados de bienes y servicios; sin embargo, en presencia de los denominados fallos de mercado, el mecanismo de precio no consigue transmitir toda la información relevante para que los agentes puedan tomar unas decisiones óptimas.

Se puede identificar seis orígenes para los fallos de mercado divididos en dos grupos (Grin, 2003): el primer grupo contempla la información imperfecta, que dificulta a los agentes económicos la toma de las decisiones correctas. En segundo lugar, la existencia de elevados costes de transacción que disuaden a los agentes de la toma de determinadas decisiones que hubieran sido beneficiosas desde el punto de vista económico. En tercer lugar podemos mencionar la inexistencia de mercados, que podría aplicarse a situaciones como la conservación de una lengua para su uso por las futuras generaciones. Por último, se encuentran las imperfecciones del mercado, como la existencia de oligopolios y monopolios, controles de tipos de cambio y tipos de interés, etc.

En un segundo grupo están las externalidades y los bienes públicos que constituyen una parte significativa del impacto de la lengua sobre la actividad económica.

La lengua genera externalidades que se encuadran dentro de las llamadas externalidades de red, estudiadas por Katz y Shapiro (1986). Existen muchos bienes que aumentan la utilidad que proporcionan a los individuos a medida que aumenta el número de personas que los utilizan. En el caso del lenguaje, cuando un individuo aprende una lengua eso confiere un beneficio a los individuos que ya la utilizan 6 .

Por su parte, Breton (1998) analiza el caso de las externalidades de red en presencia de una lingua franca. Según este autor, en ciertas ocasiones, las personas que hablan la lengua franca reciben un beneficio por este solo hecho. Breton llama a este fenómeno "señoriaje". El mismo se apreciaría al considerar a la lengua franca como una innovación tecnológica que reduce el coste de la comunicación entre individuos, lo que proporcionaría beneficios sólo a aquellos individuos que dominen dicha lengua.

Cuando un individuo aprende una segunda lengua el valor social de ese aprendizaje es mayor que el valor que representa para el individuo en términos de capital humano. Esto puede dar lugar a la presencia de fallos de mercado que justifiquen una intervención pública. En ese sentido Church y King (1993) prueban que el nivel óptimo particular (es decir, el alcanzado mediante las decisiones individuales y atendiendo sólo a sus beneficios privados) de aprendizaje de una lengua extranjera es inferior al óptimo social. La posibilidad para una acción pública que mejore el bienestar social queda abierta.

\footnotetext{
${ }^{5}$ Los tres pasos a seguir son: a) estudio de las preferencias, la demanda y la oferta, b) análisis de los diferentes mercados, c) el estudio del equilibrio general.

${ }^{6}$ Entre los estudios destacados sobre externalidades de red, podemos citar a Economides (1996), Katz y Shapiro $(1986,1994)$.
} 
El otro fallo de mercado al que nos hemos referido es el constituido por los bienes públicos. Los bienes privados tienen, entre otras características, la exclusión y la rivalidad en el consumo. Sin embargo, hay muchos bienes que no poseen estas propiedades y se denominan bienes públicos porque poseen dos propiedades fundamentales, la no exclusión y la no rivalidad en el consumo: la no exclusión significa que no puede excluirse a nadie del disfrute de un bien, aunque no pague por él y, en este caso, el coste marginal de un nuevo usuario es cero; la no rivalidad en el consumo se da cuando la utilización de un bien por parte de un individuo no reduce la capacidad de otros para utilizarlo.

Existen algunos bienes que sólo parcialmente reúnen las características de no rivalidad y no exclusión. Dentro de estos podemos citar a los bienes de club, son bienes para los cuales es posible limitar el acceso mediante tarifas, pero, una vez que se accede a los mismos, su consumo es "no rival". Para Alonso (2006), la lengua presentaría algunos rasgos de un bien de club. En primer lugar porque es un bien no rival en su consumo, y, en segundo lugar, porque, quizás exista la posibilidad de exclusión en su utilización.

Siguiendo a Jiménez (2006) podemos decir que la lengua también es un bien complejo, que puede comportarse como bien o como servicio dependiendo de la ocasión y que tiene una naturaleza dual, con características de bien privado y de bien público.

En su naturaleza de bien privado la lengua es objeto de transacciones en sí misma, como sucede en el ámbito de la enseñanza de idiomas y, además, forma parte, como un input más, de la producción de todos los bienes y servicios de un país. La lengua cumple el papel de medio de comunicación esencial para el desarrollo de la actividad económica de todos los sectores y, por último, como veremos más adelante, la lengua permite la consolidación del capital social ${ }^{7}$. En su naturaleza de bien público, cumple por una parte el principio de no rivalidad, ya que su consumo no reduce la disponibilidad de la misma. Por otra, es un bien de libre acceso, al ser imposible establecer un mecanismo que limite su consumo.

Hasta aquí hemos caracterizado la lengua en el contexto de la teoría económica convencional. Sin embargo, la lengua posee, además, características que podrían encuadrarse dentro de una categoría adicional de bienes, los llamados bienes preferentes. Esta categoría conceptual fue analizada por primera vez en 1956 por R. Musgrave, quién consideraba que los bienes preferentes son aquellos que tienen una importancia tan grande para las autoridades que sería válido modificar el resultado de la libre acción del mercado cuando el consumo de los mismos sea inferior al que desean. En este sentido, el concepto de bienes preferentes viola el principio de la soberanía del consumidor toda vez que el incremento en los niveles de provisión de un bien vaya en contra de sus preferencias, lo cual pone de manifiesto el rechazo de esta categoría de bienes por parte de la teoría económica tradicional.

\footnotetext{
${ }^{7}$ En el caso particular de algunas lenguas, como por ejemplo el español, podría darse lo que Jiménez (2006) entiende como un efecto de "prestigio" para las personas que la hablan. Este prestigio provendría no sólo de la cantidad de personas que la hablan, sino, también, de las características socioeconómicas de esa población.
} 
Una de las contribuciones más destacadas sobre el estudio de los bienes preferentes fue realizada por Ver Eecke (1998), quien considera que son bienes provistos por el Estado a unos niveles que no responden a las preferencias de los consumidores, es decir, se viola el principio de soberanía del consumidor. Ahora bien, ¿por qué motivo querría el Estado cambiar las preferencias de los consumidores? La razón más evidente es que se busca eliminar o reducir conductas dañinas para los individuos, como sucede en el caso de la legislación antitabaco o las restricciones impuestas a los menores para acceder a las bebidas alcohólicas. Existe, sin embargo, una motivación más profunda que Ver Eecke llama, a partir de Kant (2002), las condiciones para la posibilidad de la existencia o la ocurrencia de algo.

Dice Ver Eecke (1998: 139): "[...] llamaremos bienes preferentes a aquellos bienes que son las condiciones para la posibilidad de algo que es deseado por los consumidores, aun y especialmente cuando estos bienes o servicios preferentes no son preferidos por los consumidores".

Las dos claves de esta definición son, en primer lugar, la caracterización de los bienes preferentes como condiciones para la posibilidad de algo, y en segundo lugar la distinción entre el deseo de los consumidores y sus preferencias, lo que parece referirse a las diferencias que existen entre los deseos de los agentes como consumidores individuales y los deseos de los consumidores como parte de una sociedad. Un individuo puede desear fumar, pero, a la vez, considera deseable que aumente la salud de la población en general.

Llegados a este punto podríamos preguntarnos si el lenguaje puede considerarse la condición para la posibilidad de algo. Seguramente que sí: la presencia de una lengua común puede considerarse, entre otras cosas, como una precondición para la consolidación y generación de capital social.

Desde esta misma perspectiva, el lenguaje puede analizarse, como uno de los determinantes del estatus socioeconómico de las personas, de su posición dentro de un entramado social, condicionando, de esta manera, su trayectoria vital y también puede considerarse la influencia del lenguaje en el incremento de la eficiencia de las empresas.

Por último, la lengua puede considerarse como la condición para la posibilidad de una vida plena en una determinada estructura social ${ }^{8}$.

\footnotetext{
${ }^{8}$ En el debate actual sobre políticas lingüísticas surge otra función atribuida a las lenguas minoritarias y es la de constituir uno de los elementos sobre los que se pretende apoyar la potenciación de una identidad nacional y, en algunos casos, un Estado propio. Algunos autores, incluso, postulan que la lengua tiene un valor intrínseco esencial para la constitución de una comunidad. A este respecto Fishman (2001) plantea que las lenguas locales tienen una marcada función simbólica en la mayoría de las comunidades locales. La lengua, para este autor, tiene las características de un distintivo de autenticidad que reúne en sí la suma total de una experiencia histórica compartida. Además, la lengua puede ser vista como un elemento crucial para la supervivencia de una nación y su transformación en una nación-estado, Caviedes (2003).
} 


\section{EL CAPITAL SOCIAL (CS) EN UN CONTEXTO LINGÜÍSTICO}

Existen diversas aproximaciones al concepto de capital social. Como señala Portes (1998), el CS es una idea que puede retrotraerse hasta Durkheim y su énfasis en la influencia positiva de la comunidad para contrarrestar las punciones autodestructivas. Por otra parte, el CS se puede concebir considerando la distinción que hay entre una clase atomizada y una clase movilizada con capacidad para conseguir sus reivindicaciones.

Atendiendo al enfoque de este trabajo, lo más apropiado quizás sea comenzar con la definición de Bourdieu $(1986,1991)$, que distingue tres formas de capital: económico, cultural, social y simbólico. Para este autor, el capital económico es directamente convertible en riqueza material, mientras que el capital cultural, consiste en la acumulación de conocimientos y habilidades y su materialización en objetos e instituciones pero no es directamente transformable en capital económico. El capital simbólico se deriva de las otras formas de capital cuando la sociedad las reconoce como legítimas. Por último el CS es "el agregado de los recursos actuales o potenciales relacionados con la posición de una red duradera de relaciones más o menos institucionalizadas de conocimiento y de reconocimiento mutuo" (p. ).

Desde la óptica de este autor, el CS es un atributo de las clases dominantes que lo utilizan para mantenerse en el poder y conservar sus privilegios. Tiene una connotación positiva para aquellos que lo poseen, pero negativa para los excluidos y se convierte en un instrumento de dominación y de perpetuación de determinadas relaciones sociales. La adquisición de este capital responde a una estrategia social orientada de manera consciente a ese fin. Los beneficios que se obtienen de la pertenencia a un grupo son la base de la solidaridad dentro del mismo que, a su vez, hace posible la existencia de dichos beneficios. Como señala Field (2003), el tratamiento que hace Bordieu del CS es, en cierta manera, circular: determinados individuos mantienen sus privilegios utilizando las conexiones que conforman su capital social para interactuar con otros individuos de su misma clase.

Como se verá más adelante, la concepción de Bourdieu tiene un interés especial para este trabajo, ya que el CS puede tener efectos negativos (en su caso favoreciendo a una clase social determinada en detrimento del conjunto de la sociedad). Si bien plantearemos que, en algunos casos concretos, la existencia de redes y relaciones dentro de un grupo puede perjudicar a los que forman parte del mismo, al debilitar sus conexiones con el entorno de dicho agregado.

En este sentido, un enfoque más cercano al de este trabajo, en lo que respecta a la relación de los grupos minoritarios con el capital social, es el de Coleman (1988, 1990). Este autor extiende la posesión y disfrute del capital social a los grupos marginales de la sociedad, como pueden ser los colectivos de inmigrantes. Para Coleman, el CS no es una sola entidad sino una variedad de diferentes formas que tienen dos características en común: todas consisten en algunos aspectos de la estructura social y, además, facilitan ciertas acciones de los individuos que están dentro de la estructura. 
Según este invetigador, el CS tiene dos vertientes, la individual y la colectiva. La primera de ellas se manifiesta en la integración social del individuo, en ella tiene un lugar importante su red de contactos sociales. La segunda vertiente está representada por la existencia de una atmósfera de confianza y seguridad en la sociedad y reciprocidad de comportamiento en las interacciones entre personas ${ }^{9}$.

EI CS se puede presentar de tres maneras; en primer lugar, obligaciones y expectativas que dependen de la fiabilidad del entorno social; en segundo lugar, la facilidad con la que fluye la información a través de la estructura social y, finalmente, en tercer lugar, la existencia de normas acompañadas de sanciones efectivas cuando no se cumplen. Coleman, en ciertos aspectos, tiene una concepción del capital social completamente contrapuesta a la de Bordieu. El conjunto de normas y sanciones que éste plantea permite que los individuos cooperen entre sí, de manera que se obtengan ventajas individuales, para un colectivo determinado y para la sociedad en su conjunto. No sucede, como en el caso de Bourdieu, que la ganancia de un colectivo determinado, derivado de la posesión de ese capital social, se transforma en pérdidas para otros colectivos o para la sociedad en su conjunto.

Otra aproximación al concepto de CS es la de Putnam (2000). Para Putnam mientras que el capital físico se refiere a los objetos físicos y el capital humano hace mención a las cualidades de los individuos, el CS hace referencia a las conexiones entre los individuos, las redes sociales y las normas de reciprocidad que emergen de ellas ${ }^{10}$. Según este autor, el capital social tiene la capacidad de favorecer, de garantizar que la sociedad se guíe de acuerdo con unas normas de comportamiento que se consideren deseables. Además, el CS promueve la confianza y la reciprocidad en la interacción entre los miembros de la sociedad. Esto es un resultado deseable, ya que una sociedad caracterizada por la existencia de una reciprocidad generalizada es más eficiente que una sociedad donde esto no se produce.

Putnam recoge la existencia de dos tipos de vínculos entre las personas, los bridging, que se establecen entre personas de diferentes clases sociales y los bondging que se dan entre iguales, entre miembros de grupos homogéneos, como, por ejemplo, una familia. En relación con las ventajas derivadas de la existencia del capital social para una comunidad, plantea las siguientes: en primer lugar, la existencia de confianza y redes sociales dentro de una comunidad pueden tener efectos beneficiosos desde el punto de vista económico que permitan mitigar situaciones socioeconómicas desfavorables. En segundo lugar, Putnam sostiene que aquellas áreas que tienen un mayor CS, constituyen un entorno mucho más

\footnotetext{
${ }^{9}$ El capital social aumenta la confianza entre los individuos, lo que facilita su interacción y promueve la creación de redes sociales.

${ }^{10}$ Collier (1988) entiende por CS la coherencia interna, social y cultural, de la sociedad, las normas y valores que rigen la interacción entre las personas y las instituciones que subyacen a sus acciones. La confianza, la reciprocidad, las redes interpersonales, la cooperación y la coordinación entre las personas pueden ser vistas como una forma de CS que reporta externalidades a la sociedad.
} 
favorable para el crecimiento de los niños, tanto por una menor tasa de criminalidad, como por la existencia de confianza y redes y normas de reciprocidad. Finalmente, este autor extiende los beneficios del CS a la salud; así, según su criterio, aquellos que participan en la actividad de distintos grupos sociales pueden ver incrementada su calidad y esperanza de vida de una manera significativa.

EI CS derivado de vínculos de tipo bondging, como plantea Granovetter (1973), puede tener un papel importante en la movilidad geográfica de las personas, pero también puede provocar un efecto de exclusión, es decir, limitar su movilidad social. En ese sentido, los vínculos fuertes (bondging) permiten mantener a los individuos en armonía con su grupo, bajo su protección, mitigando las agresiones del exterior; los vínculos débiles (bridging) permiten avanzar y relacionarse con personas de círculos exteriores al suyo, abrirse y situar su campo de acción en una escala superior a la de su círculo más cercano.

En el caso de los inmigrantes, los vínculos fuertes cumplen un papel mucho más complejo que en el resto de la sociedad. Cuando los inmigrantes llegan al país de acogida, en la mayoría de los casos, pasan a formar parte del sector informal de la economía. Sus compatriotas, en muchos casos de la misma zona geográfica o, incluso del mismo pueblo, les ayudan a conseguir empleo, generalmente en el sector en el que ellos trabajan, les facilitan encontrar vivienda e, incluso, les proporcionan capital para empezar un negocio propio (lo que es de especial relevancia para personas que están, generalmente, excluidas del sistema financiero tradicional). Una manifestación de este comportamiento la encontramos en las grandes ciudades europeas, donde los inmigrantes de una determinada nacionalidad copan una rama de actividad o se concentran en una determinada zona. Ahora bien, esta red de apoyo que espera a los inmigrantes en el país de destino constituye un CS que los mismos traen desde sus países de origen. Evidentemente su existencia facilita, y mucho, la supervivencia de los inmigrantes en la sociedad de acogida y, hasta cierto punto, su integración en la misma. Este tipo de comportamiento se refleja en dos conceptos teóricos, el de los enclaves étnicos de negocios y los nichos étnicos (Portes, 1988).

Los enclaves étnicos corresponden a concentraciones densas de empresas cuyos propietarios son inmigrantes y que emplean en una proporción significativa la mano de obra de sus compatriotas, desarrollando una presencia física distintiva en el espacio urbano. Un nicho étnico aparece cuando un grupo se hace con un sector determinado de actividad, de tal manera que sus miembros tienen acceso privilegiado a los empleos de dicho sector o a las nuevas aperturas de negocios. Además actúan restringiendo la entrada de todos aquellos que no pertenecen al grupo ${ }^{11}$.

Por último queda apuntar dos cuestiones sobre el CS: la primera tiene que ver con la forma de medir el CS y la segunda tiene que ver con la forma de analizar este concepto en un contexto de desarrollo y subdesarrollo.

\footnotetext{
${ }^{11}$ Algunos estudios recogen la influencia del país de origen del inmigrante sobre su posición en el mercado laboral. Podemos citar a Miller (1987) y Satzewich y Li (1987).
} 
En cuanto a la forma de medir el CS, no hay acuerdo al respecto, pero las primeras aproximaciones apuntan a un cálculo vía el residual del capital físico y el capital natural. En este sentido, el Banco Mundial (2005) ha realizado un esfuerzo considerable por aclararnos este problema y ensaya alguna estimación del CS donde se muestra que su valor es muy importante a la hora de abordar el análisis de la riqueza y bienestar de una sociedad. Quedaría por tanto avanzar en una línea de trabajo que indique cuánto de ese CS está influenciado por la lengua.

Respecto a la segunda cuestión, hay que indicar que cuando se aborda la problemática del CS es útil diferenciar el contexto: en uno desarrollado la forma de abordar una medida del beneficio del CS es a través de la reducción de los costes de transacción, mientras que esto no es relevante en un contexto subdesarrollado, pues la reducción de los costes de transacción no son relevantes (debido a que el proceso productivo es mucho más simple) y en su lugar la cuestión pasa por considerar los beneficios que proporciona una red de protección social frente a los costes que, en muchos casos, implica la exclusión de quien no pertenece al grupo.

\section{LOS EFECTOS DEL LENGUAJE SOBRE EL ESTATUS SOCIOECONÓMICO DE LAS PERSONAS}

Siguiendo a Coleman (1988), podemos identificar alguno de los canales a través de los cuales se producen los efectos beneficiosos de la pertenencia a un grupo. El primero de ellos es el aumento de la transmisión de información entre sus miembros, información que puede referirse a los miembros del grupo 0 a personas ajenas al grupo (esto haría más eficientes las relaciones económicas). Este efecto es particularmente importante en el caso de inmigrantes que no hablan la lengua del país de destino y sirve, como comentamos en la sección anterior, para transmitir información sobre oportunidades de empleo, vivienda, sanidad, derechos, etc. El compartir información relevante, desde un punto de vista económico, genera una externalidad positiva.

Como ejemplo de esta externalidad positiva en el caso del lenguaje, podemos considerar los posibles efectos de red en el acceso al sistema de protección social en un país receptor de inmigración (Bertrand et al., 2000). Analizando datos de Estados Unidos, estos autores consideran la hipótesis de que los miembros de las comunidades de inmigrantes de habla no inglesa residentes en ese país tienden a obtener la mayoría de la información sobre el sistema de seguridad social de personas que pertenecen a su mismo grupo lingüístico. Se plantea la existencia de efectos de red entre los inmigrantes. La interacción entre los inmigrantes que hablan una misma lengua proporciona a estas personas la posibilidad de acceder a una información antes vetada para ellos y, por tanto, la posibilidad de obtener unos beneficios sociales de los que, en otras circunstancias (de no presentarse estas externalidades de red) no gozarían.

Sin embargo, y como aspecto negativo, los inmigrantes que pertenecen a estos grupos reciben menos información sobre los empleos disponibles en la sociedad de acogida y, por 
tanto, este factor contribuye a que tengan menores probabilidades de encontrar empleo que el resto de la población. En realidad, cabe pensar que no es la información sobre los puestos de trabajo lo que falla, sino que se genera en este grupo una tendencia a la especialización en la obtención de ayuda pública.

Una gran parte del coste de acceder a este tipo de recursos proviene de la necesidad de obtener la información necesaria sobre el funcionamiento de la administración pública y sobre las ayudas disponibles. Una vez que el colectivo posee esta información, el coste para sus miembros es casi marginal. La decisión de acudir a las ayudas públicas en vez de buscar un empleo en el sector privado se convierte, entonces, en perfectamente racional desde el punto de vista económico.

En el artículo de Bertrand et al., (2000) los autores realizan un estudio econométrico utilizando el lenguaje hablado en el hogar como una variable proxy de los lazos sociales existentes entre los individuos dentro de una vecindad. Los autores asumen que las personas que residen en una zona donde vivan más personas que hablen su mismo idioma tendrán acceso a una mayor cantidad de contactos. Como variable que mida la cantidad de redes que se pueden formar, $\mathrm{o}$, dicho de otra manera, la disponibilidad de contactos en una zona determinada, se elige el número de personas en un área que hable el idioma objeto del estudio. Los contactos que correspondan a personas que usan en mayor medida los sistemas de protección social tendrán el efecto de facilitar, a quien posee dichos contactos, el acceso a este tipo de prestaciones. A partir de aquí, los autores plantean que el diferente acceso a las prestaciones sociales de los diferentes grupos lingüísticos nos dará una medida de la diferente calidad de las redes que se establecen dentro de esos grupos. La conclusión que parece extraerse de este trabajo es que la pertenencia a uno de estos grupos maximiza la posibilidad de supervivencia en un entorno nuevo pero, a la vez, podría tener el efecto no deseado de aumentar la dependencia de estos colectivos de la protección social ${ }^{12}$.

Un segundo canal por el que actúa el capital social de manera positiva, sobre los miembros de un grupo es mediante la construcción de una identidad común, la existencia de esta identidad puede paliar el desarraigo pero, a la vez, puede dificultar su integración en la sociedad de acogida. Finalmente, la coordinación explícita entre los miembros de un grupo permite acciones colectivas encaminadas a socorrer a los miembros que pasan apuros 0 en defensa de determinados derechos.

Sin embargo, lo que es positivo para estos inmigrantes en un primer momento, puede no serlo tanto, o por lo menos tener repercusiones negativas ${ }^{13}$ en los planos económico y social en un momento posterior 0 , sobre todo, en el caso de sus hijos, nacidos en el nuevo

\footnotetext{
${ }^{12}$ Se utilizan datos del censo de Estados Unidos de 1990, los datos estudiados corresponden a 42 grupos lingüísticos, más del $50 \%$ de los sujetos estudiados hablaba español.

${ }^{13}$ La visión de Coleman (1988) no recoge estos efectos negativos del capital social, ya que, para este autor, la presencia del mismo y del sistema de normas y sanciones permitiría que los individuos establecieran una colaboración beneficiosa para todos ellos. En este sentido, veremos cómo, bajo nuestro enfoque, aparecerían algunas connotaciones negativas del capital social, relacionadas, de una manera semejante a lo que sucede bajo la óptica de Bordieu, con situaciones de exclusión social.
} 
país. Estos efectos negativos tienen su origen en las dificultades que puede provocar, a la hora de interrelacionarse con la sociedad en la que están insertos, el estar imbricado en un entramado de relaciones con sus compatriotas, tan fuerte y, hasta cierto punto, excluyente. Este alejamiento de la sociedad de acogida es mayor cuanto menor sea el dominio, por parte de los inmigrantes, del idioma del país de destino.

Algunos trabajos, englobados dentro de la llamada economía del lenguaje, abordaban el estudio de la lengua centrándose en los efectos que tenía sobre el estatus socioeconómico el que los individuos hablaran distintas lenguas. En general estos estudios siguieron dos direcciones, por una parte, analizaron las repercusiones salariales que tiene el aprendizaje de una segunda lengua, enfocando el problema desde el punto de vista de un incremento del capital humano. Por ejemplo Grin (1995a, 1995b) encuentra que, en el caso de Suiza, el conocimiento de idiomas conlleva un incremento significativo de salarios entre los hombres, pudiendo llegar a un rango del $13,6 \%$ al $16 \%$ en el caso de conocimiento del inglés. Grin y Vaillancourt (1987), en un estudio realizado para Canadá, encuentran incrementos salariales de entre un $5 \%$ y un 10\%. Por otra parte Chiswick y Miller (1995) consideran que el conocimiento de la lengua tiene las tres características básicas del capital humano: en primer lugar, no se puede separar de la persona que lo posee, en segundo lugar, aumenta la productividad del sujeto y, finalmente, se adquiere a costa de un sacrificio de tiempo y dinero.

Por otra parte, un segundo grupo de estudios se acercaron al análisis de la repercusión sobre el estatus socioeconómico de los inmigrantes que tiene el hablar lenguas distintas a la de su país de acogida. Si bien muchos trabajos de este último grupo abordan también el problema desde la perspectiva del capital humano, es evidente que la profundidad de la cuestión excede este marco y se obtiene un análisis más completo si se enfoca el problema desde la óptica del capital social. Así encontramos trabajos como los de Ruggler y Sobek (1997) que analizando la situación de los inmigrantes mexicanos en Estados Unidos, concluyen que los sueldos de los inmigrantes son, en promedio, un $50 \%$ inferior a los de los nativos. Chiswick y Miller (1999) plantean una explicación cercana al capital social para esas diferencias salariales. Según estos autores, en el año 1992 un $42 \%$ de la población hispana de Estados Unidos vivía en barrios predominantemente hispánicos ${ }^{14}$. Los motivos que explicarían esta concentración de inmigrantes en determinados barrios pueden ser varios, aunque seguramente no es una motivación menor el sentirse cómodo y aceptado en un entorno de personas que comparten una misma lengua y cultura. Esto es lo que plantea Field (2003) cuando postula que el sentido de pertenencia a una comunidad y el disfrutar de las redes sociales que ésta ofrece a sus miembros le proporciona a los individuos diversos beneficios, entre los que se encuentra el sentimiento de bienestar.

Por otra parte, los escasos ingresos de los inmigrantes no les permiten optar a residir en barrios más caros. Esta situación tiene el potencial de autoperpetuarse a lo largo del

\footnotetext{
${ }^{14}$ Es interesante remarcar que el estudio habla de barrios hispanos; como ya hicimos notar, el idioma puede convertirse en un aglutinante tanto o más importante que la procedencia.
} 
tiempo ${ }^{15}$ ya que se estaría conviviendo con personas que, por término medio, tienen un bajo nivel educativo, escasos conocimientos de inglés y una baja capacidad para generar ingresos. Estos factores contribuirían a la formación de zonas económicamente deprimidas en las cuales es difícil que se creen oportunidades económicas para sus habitantes.

Por el mismo hecho de vivir en esas zonas, se está favoreciendo la continuidad de una situación de exclusión social y se podría enmarcar en lo que Putnam (2000) llama los vínculos fuertes. Para este autor, el capital social basado en este tipo de relaciones es más introspectivo, los miembros del grupo son más proclives a relacionarse entre ellos en detrimento de las relaciones con el exterior, lo que provoca una tendencia al fortalecimiento de identidades exclusivas y grupos homogéneos. Por el contrario, los vínculos débiles tienden a provocar, una dinámica más abierta, con una mayor tendencia a la relación con personas de otros grupos distintos.

Los resultados del estudio de Chiswick et al., (2000) para Bolivia van en esta dirección: presencia de discriminación salarial en contra de las mujeres que hablaban alguna de las lenguas indígenas además de español. En concreto, aquellas que hablaban solamente español obtenían un salario un $22 \%$ superior al de las mujeres que hablaban una de las lenguas indígenas junto con el español, y estas últimas, a su vez, obtenían unos ingresos un $25 \%$ superiores al de aquellas mujeres que hablaban exclusivamente lenguas indígenas. De más está decir que este dato puede estar segado por la existencia de discriminación racial y cabe recordar que en este trabajo intentamos analizar la influencia de la lengua sobre el capital social y las repercusiones ulteriores de esta relación. Sin embargo, el que las mujeres que hablen español junto con una lengua indígena obtengan unas retribuciones un $25 \%$ superiores al de aquellas mujeres que hablan sólo estas últimas, podría indicar, no sólo una diferencia de capital humano, sino también una diferencia de capital social, una diferencia de redes, de contactos, de interrelaciones, al igual que sucedía en el caso de los inmigrantes que llegaban a un país sin hablar el idioma de éste ${ }^{16}$.

Otros estudios, como el de Raynauld y Marion (1972), parecen confirmar esta tendencia. Para estos autores, las distintas lenguas maternas dividen a los individuos en grupos, lo que se traduce en diferencias en su estatus socio-económico. Esto se producirá, sobre todo, cuando exista una discriminación deliberada por parte de los miembros de un grupo dominante. En esta línea también se pueden citar los trabajos de Fogel (1966), Grin (1997) y Grin y Sfreddo (1998). Todos ellos encuentran evidencias empíricas de la existencia de diferencias salariales entre personas que pertenecen a grupos lingüísticos distintos.

\footnotetext{
${ }^{15}$ En este sentido, al igual que sucede en la concepción de Bordieu, el capital social tendría connotaciones negativas para un colectivo, si bien para este autor los efectos negativos los sufrirían quienes no lo poseen y en este caso los perjudicados serían los poseedores.

${ }^{16}$ Existen otros estudios que realizan estimaciones empíricas del valor que representa la adquisición de la lengua dominante de una región. Entre estos se pueden citar a Grenier (1984), Chiswick y Miller (2002), Dávila y Mora (2000), Dustmann y Van Soest (2001).
} 


\section{EL LENGUAJE, LAS EMPRESAS Y EL CAPITAL SOCIAL}

En este apartado nos centraremos en los efectos de la lengua sobre la eficiencia de las empresas. Partimos de asumir, de acuerdo con lo expuesto en los apartados anteriores, que el lenguaje tiene repercusiones sobre el capital social de los agregados humanos. Por tanto, los canales a través de los cuales el capital social produce una mejora en la eficiencia de un colectivo actúan como correa de transmisión de la influencia del lenguaje dentro de las empresas. A efectos de nuestro análisis, podemos considerar los siguientes canales mediante los cuales se ejerce la influencia del capital social: en primer lugar, la mejora en la difusión de la información, lo que da lugar a una reducción de las asimetrías de información y una disminución de los costes de supervisión y transacción. En segundo lugar, el capital social contribuye a la creación de una identidad de grupo, lo que mitiga el efecto de la existencia de incompatibilidades de incentivos entre miembros del colectivo.

A este respecto, Fershtman y Gneezy (2001), a partir de experimentos utilizando teoría de juegos, plantean que las personas tienden a comportarse de una manera más cooperativa y exhiben un mayor grado de altruismo, lo que lleva a un resultado más eficiente, si se identifican con un grupo. Esta situación se da, incluso, cuando los miembros del grupo son desconocidos y ni siquiera se tiene contacto con ellos durante el experimento. La identificación con el grupo parece ser la clave para que suceda. Como sugieren los autores, si la identificación con un grupo es necesaria para que los individuos se comporten de una manera más cooperativa y altruista con los restantes miembros del mismo, entonces los esfuerzos para crear o reforzar ese sentimiento de pertenencia al grupo constituyen un elemento primordial del capital social.

Es fácil ver como la lengua, sobre todo en el caso de inmigrantes inmersos en un idioma distinto al suyo se constituye en un catalizador de primer orden a la hora de fomentar la aparición de la identificación con el grupo. Se puede considerar, incluso, que la lengua es un aglutinante más importante que la nacionalidad de origen. Esto se ve sobre todo en el caso de inmigrantes que proceden de una misma zona lingüística, por ejemplo los inmigrantes latinoamericanos en países de habla inglesa, los cuales tienen un primer círculo de relaciones con sus compatriotas y otro inmediatamente contiguo a este primero en el que se relacionan con personas que hablan su mismo idioma, procedan 0 no del mismo país.

Finalmente, un tercer canal es el establecimiento de normas colectivas de comportamiento, lo que da un marco estable a las relaciones entre los miembros del grupo y mitiga las fricciones derivadas de las interacciones entre los mismos.

Algunos estudios que analizan el fenómeno de la lengua dentro de las empresas son útiles para ilustrar estos canales de transmisión. Podemos citar el trabajo de Migué (1970), que analiza las dificultades con las que se encuentran, a la hora de acceder a un empleo, aquellos trabajadores que no hablan la lengua de sus empleadores. Este autor encuentra que, como cabría esperar, los empleadores prefieren contratar trabajadores que hablen su mismo idioma. Las explicaciones para este comportamiento son, por una parte, que la proximidad cultural, representada por el dominio de la misma lengua permitiría que los 
empleadores evaluaran más acertadamente la productividad de sus futuros empleados que en el caso contrario. Es decir, el lenguaje conseguiría reducir las asimetrías de información, reduciendo los costes de supervisión y, lo que es más importante, no es sólo el lenguaje compartido como código de comunicación lo que reduce estos costes, sino también la cultura compartida que se presupone por hablar un mismo idioma. El lenguaje se convierte en la base sobre la que se construyen unos códigos de conducta compartidos, es decir, sobre la que se articula el crecimiento del capital social dentro de la empresa. Claro está que la ganancia más importante, y la más obvia, de productividad procede de la eliminación de las dificultades de comunicación dentro de la empresa que supondría la convivencia de personas que hablaran diferentes idiomas.

En esta dirección, Lang (1986) explica las diferencias salariales entre grupos que hablan diferentes lenguas como consecuencia de las dificultades de comunicación entre patronos y empleados y los costes que ello conlleva dejando de lado la existencia de algún tipo de discriminación por el origen de los trabajadores.

En el caso de empresas multinacionales que desarrollan su actividad en distintos países, en alguno de los cuales se hablan varias lenguas dependiendo de la región, la lengua institucional no sólo tiene el efecto de facilitar la comunicación entre los empleados, reduciendo costes, sino que también sirve como vehículo de una serie de valores que se consideran propios de la empresa. Supongamos, por ejemplo, el caso de una multinacional alemana asentada en un país como Suiza: el idioma alemán no sólo, en este caso, es el idioma de la matriz, sino que es también el hablado por una parte importante de la población del país receptor ${ }^{17}$. El que todos los empleados de la empresa, independientemente de la zona, hablen alemán, aumenta la productividad, no sólo por los canales evidentes de la disminución de las asimetrías de información y de los costes de supervisión y transacción, sino que contribuye también a la creación de una identidad de grupo dentro de la empresa y, además, el hecho de que se considere deseable que los empleados hablen alemán constituye una norma colectiva de comportamiento. Con una simple medida como promover el conocimiento de la lengua de la casa matriz, se produce el efecto de incrementar el capital social de la empresa con el consiguiente incremento de la productividad que ello trae aparejado. Sin tener en cuenta, claro, las ganancias en términos de eficiencia en la relación entre la empresa filial y la central ${ }^{18}$.

\footnotetext{
${ }^{17}$ Aproximadamente un $70 \%$ de la población suiza habla alemán, el francés es hablado por un $20 \%$, el italiano por un $10 \%$ y el romanche por el $1 \%$.

${ }^{18}$ Estas empresas se diferencian por las siguientes características: gran coordinación internacional de la producción y baja independencia de los mercados. Mercado de carácter global, no existen productos especíicos para cada mercado estatal. La gestión se fundamenta en criterios correspondientes al país de origen de la corporación. Identidad caracterizada por unos valores, un idioma y un personal directivo que comparten una misma nacionalidad. Las decisiones relevantes se toman en la matriz. Coordinación global, elevada movilidad de directivos. El acceso a la clase directiva se alcanza tras largos períodos de estancia en la matriz, una vez dominado el idioma corporativo. El idioma corporativo adquiere valor como manifestación de poder e identidad.
} 
Dhir y Savage (2002) plantean que el lenguaje de una organización puede ser visto como el depositario de su conocimiento. Podemos ir un paso más allá y decir que el lenguaje dentro de una organización cumple también el papel de receptáculo de su esencia, su espíritu, su cultura.

Este tipo de compañías corresponderían a lo que Sole et al. (2005) llaman compañías etnocéntricas. Frente a éstas están las llamadas compañías geocéntricas que al contrario de las anteriormente nombradas, cuyo funcionamiento provoca el incremento del capital social dentro de la empresa, valora más el conocimiento por parte de los empleados y de los idiomas de los Estados receptores. Con ello se pretende disfrutar del capital social de la sociedad de acogida, del conocimiento de normas y costumbres que faciliten la interacción con este mercado. El estudio de Solé et al. (2005) toma como referencia a las empresas instaladas en Cataluña. En él se analizan los problemas de eficiencia y distribución de los recursos dentro de dichas empresas, tomando como base criterios étnico-lingüísticos. En este trabajo se plantean distintos escenarios empresariales que se diferencian en el grado de movilidad de los factores de producción. Posteriormente se analizan los regímenes lingüísticos de los mismos y los criterios lingüísticos de selección y promoción del personal.

La hipótesis principal de esta investigación es que las elecciones lingüísticas de los individuos ${ }^{19}$ dependen de su posición laboral en las empresas, de las elecciones corporativas $^{20}$ de su estructura organizativa y de su posición en la competencia mundial. Una de las cuestiones a las que se enfrentan las empresas, dentro de este marco de análisis, es la identificación de las circunstancias adecuadas que aconsejan la adopción de una lengua franca internacional para las comunicaciones internas y el abandono del idioma tradicional dominante, asociado al origen nacional de la empresa. Es decir, el abandono del idioma de los propietarios y la alta dirección a favor de otros idiomas presentes en la organización.

Se postulan, entonces, dos estrategias lingüísticas diferenciadas: en primer lugar, el reforzamiento de las divisiones lingüísticas como mecanismo de segmentación de los recursos y, en segundo lugar, se busca aumentar la eficacia y la eficiencia corporativa, accediendo a clientes lingüísticamente heterogéneos y reduciendo los costes de transacción internos por medio de la desetnificación del idioma corporativo.

Los resultados obtenidos en este estudio muestran que, en los diferentes escenarios con los que trabaja, se produce una importante desigualdad en la promoción o en la selección de los trabajadores, en función del capital lingüístico de cada uno. Se produce una estratificación ocupacional de acuerdo con los grupos lingüísticos a los que pertenezcan los trabajadores. Además, otra conclusión importante a la que se llega es que el origen del capital de la empresa constituye una variable clave a la hora de entender las relaciones

\footnotetext{
${ }^{19} \mathrm{Es}$ decir, la lengua con la que intentarán comunicarse con su entorno y sus decisiones con respecto al aprendizaje de nuevos idiomas.

${ }^{20}$ Las elecciones corporativas comprenden, por ejemplo, la utilización de una lengua franca dentro de la organización y la diversidad lingüística que se considera apropiada para la empresa dentro de un país o a nivel internacional.
} 
que se establecen, dentro de ella, entre los diferentes grupos lingüísticos. Por otra parte, los autores llegan a una conclusión mencionada anteriormente: el idioma de la propiedad de la empresa transmite la cultura de la misma, de tal manera que los idiomas se constituyen en un mecanismo de control en el acceso a los distintos escalafones laborales.

\section{Consideraciones finales}

A lo largo de este trabajo se puede observar como el lenguaje, entendido como un bien preferente, tiene una influencia significativa en la aparición del llamado capital social en los agregados humanos. Se reconocen tres canales por los cuales dicho capital tiene efectos beneficiosos sobre los individuos. El primero de ellos es el aumento de la transmisión de información entre sus miembros. El compartir información relevante, desde un punto de vista económico, genera una externalidad positiva. Como ejemplo de esta externalidad positiva en el caso del lenguaje hemos citado los posibles efectos de red en el acceso al sistema de protección social en un país receptor de inmigración. Un segundo canal por el que actúa el capital social de manera positiva sobre los miembros de un grupo es mediante la construcción de una identidad común; la existencia de esta identidad puede paliar el desarraigo pero, a la vez, puede dificultar su integración en la sociedad de acogida.

Finalmente, la coordinación explícita entre los miembros de un grupo permite acciones colectivas encaminadas a socorrer a los miembros que pasan apuros 0 en defensa de determinados derechos. El capital social puede tener un papel positivo importante en la movilidad geográfica de las personas pero puede provocar también un efecto de exclusión. Diversos estudios indican que las lenguas maternas dividen a los habitantes de un país en grupos, lo que tiene su reflejo en diferencias en sus estatus socioeconómico.

En el caso de las empresas, el lenguaje puede tener efectos positivos sobre la eficiencia a través de una reducción de las asimetrías de información y una disminución de los costes de supervisión y transacción. Un tercer canal es el establecimiento de normas colectivas de comportamiento, lo que da un marco estable a las relaciones entre los miembros del grupo y mitiga las fricciones derivadas de las interacciones entre los mismos. Diversos estudios muestran que dentro de las empresas se produce una importante desigualdad en la promoción o en la selección de los trabajadores, en función del capital lingüístico de cada uno; además, el idioma de la propiedad de la empresa transmite la cultura de la misma, de tal manera que los idiomas se constituyen en un mecanismo de control en el acceso a los distintos escalafones laborales.

En definitiva, la relación entre lenguaje y capital social mejora nuestra comprensión de la forma en que los individuos y la sociedad pueden mejorar su bienestar a partir de una idea de riqueza (capital social) que no siempre se trata de forma explícita y que se complementa con el capital físico, humano, natural y cultural. 


\section{Referencias BibliográfícAs}

Alonso, J. A. 2006. Naturaleza económica de la lengua. Instituto Complutense de Estudios Internacionales. Documento de Trabajo 02/06.

Bertrand, M., E.F. Leuttmer y S. Mullainathan. 2000. «Network effects and welfare cultures». Quarterly Journal of Economics 115: 1019-1056.

Bourdieu, P. 1986. "The Forms of Capital". En Richardson, J.G. (ed.) Handbook of Theory and Research for the Sociology of Education, pp. 241-258. New York: Greenwood Press.

Bourdieu, P. 1991. Language \& Symbolic Power. Cambridge. Massachusetts: Harvard University Press.

Breton, A. 1998. "An economic analysis of language", en Breton, A. (ed.), Economic Approaches to Language and Bilingualism, pp. 1-33. Ottawa: Canadian Heritage.

Caviedes, A. 2003. "The Role of Language in Nation-Building within the European Union". Dialectical Antropology 27: 249-268.

Chiswick, B. y P. Miller. 1995. "The Endogeneity between Language and Earnings: International Analyses". Journal of Labor Economics 13: 246-288.

Chiswick, B y P. Miller. 1999. "Language Skills and Earnings among Legalized Aliens". Journal of Population Economics 12: 63-89.

Chiswick, B., H.A. Patrinos y M. Hurst. 2000. "Indigenous language skills and the labor market in a developing economy: Bolivia". Economic Development and Cultural Change 48: 349-67.

Chiswick, B y P. Miller. 2002. "Immigrants earnings: Language skills, linguistic concentrations and the business cycle". Journal of Population Economics 15: 31-57.

Church, J. y I. King. 1993. "Bilingualism and network externalities". Canadian Journal of Economics 24: 337-345.

Coleman, J. 1988. "Social Capital in the Creation of Human Capital". American Journal of Sociology 94: 95-210.

Coleman, J. 1990. Foundations of Social Theory. Cambridge Mass.: Harvard University Press.

Collier, P. 1998. "Social Capital and Poverty". World Bank Social Capital Initiative Working Paper 4. Washington, D.C.

Dávila, A. y M. Mora. 2000. "English fluency of recent Hispanic immigrants to the Unites Status in 1980 and 1990". Economic Develooment and Cultural Change 48: 369-89.

Dhir, K. S. y T. Savage. 2002. "The Value of a Working Language". International Journal of the Sociology of Language 158: 1-35.

Dustmannu, C. y A. Van Soest . 2001. "Language fluency and earnings: Estimation with misclassified language indicators". Review of Economic and Statistics 83: 663-74. 
Economides, N. 1996. "The Economics of Networks". International Journal of Industrial Organization 14: 673-699.

Fershtman, C. y U. Gneezy. 2001. "Discrimination in a segmented society: An experimental approach". Quarterly Journal of Economics 116: 351-77.

Field, J. 2003. Social Capital. Londres: Routledge.

Fishman, J. A. 2001. "El nuevo orden lingüístico", Digithum revista digital d'humanitats, 3). Available at: http:// www.uoc.edu/humfil/articles/esp/fishman/fishman.html. Retrieved on January 15th, 2009.

Fogel, W. 1966. "The effects of low educational attainment on incomes: A comparative study of selected ethnic groups", Journal of Human Resources 1: 22-40.

Granovetter, M. 1973. Getting a Job. A Study of Contacts and Careers, Chicago: University of Chicago Press.

Grenier, G. 1984. "The effects of language characteristcs on the wages of Hispanic-American males". Journal of Human Resources 19: 22-40.

Grin, F. 1994. "The economics of language: match or mismatch?". International Political Science Review 4: 27-44.

Grin, F. 1995a. "La Valeur des Compétences Linguistique: Ver Une Perspective Économique». Babylonia 2: $59-65$.

Grin, F. 1995b. «La valeur privée de la pluralité linguistique». Seminario Mercator: 'Approches économiques aux langues minoritaires'. Maisondes Sciences de l'Homme: París.

Grin, F. 1997. Language et Differentiels de Statut Socio-économique en Suisse. Berne: Federal Statistical Office.

Grin, F. 2003. "Language Planning and Economics". Current Issues in Language Planning 4: 1-66.

Grin, F. y C. Sfreddo. 1998. "Language-based earnings differentials on the Swiss labour market: Is Italian a liability?". International Journal of Manpower 19: 520-32.

Grin, F. y F. Vaillancourt. 1997. "The economics of Multilingualism: Overview of the Literature and Analitical Framework". En Grave, W. (ed.). Multilingualism and Multilingual Communities, pp. 43-65. Cambridge: Cambridge University Press.

Jiménez, J. C. 2006. La Economía de la lengua: una visión de conjunto, ICEI, Documentos de trabajo 01/06.

Kant, I. 2002. La crítica de la razón práctica. Madrid: Alianza Editorial.

Katz, M. L. y C. Shapiro. 1986. "Technology Adoptiv in the Presence of Network Externalities". Journal of Political Economy 94: 822-841.

Katz, M. L. y C. Shapiro. 1994. "System Competition and Network Effects". Journal of Economic Perspective 8: 93-116.

Lang, K. 1986. "A language theory of discrimination". Quaterly Journal of Economics 101: 363-82. 
Migué, J.L. 1970. «Le nationalisme, I'unité antionale et la théorie économique de línformation. Revue Canadienne d'Economie 3: 183-98.

Miller, P.W. 1987. «Aspects of occupational mobility and attainment among immigrants in Australia". International Migration Review 21: 96-109.

Portes, A. 1998. "Social Capital: Its Origin and Application in Modern Sociology". Annual Revenue of Sociology 24: $1-24$.

Putnam, R.D. 2000. Bowling Alone. The Collapse and Revival of American Community. Nueva York: Simon and Schuster.

Raynauld, A. y P. Marion. 1972. «Une analyse économique de la disparité inter-ethnique des revenues». Revue Économique 23 : 1-19.

Ruggles, S. y M. Sobek et al. 1997. «Integrated Public Use Microdata Series: Version 2.0. www.ipums.org, Minneapolis. Historical Census Projects: University of Minnesota.

Satzewich, V. y P.S. Li. 1987. "Immigrant labour in Canada: The cost and benefits of ethnic origin in the job market". Canadian Journal of Sociology 12: 229-241.

Solé, C., A. Alarcón, A. Terrones y L. Garzón. 2005. "Eficiencia y discrimación lingüística en la empresa". Revista Española de Investigaciones Sociológicas 109:11-35.

Ver Eecke, W. 1998. "The Concept of a Merit Good”. Journal of Socio-Economics 27: 133-153.

Vaillancourt, F. 1985. Economie et langue. Conseil de la Langue Francaise: Québec.

World Bank. 2005. Where is the Wealth of Nations?: Measuring Capital for the XXI Century. Conference Edition. Washington D.C., July 15, 2005. Http://siteresources.worldbank.org/INTEEI/Home/20666132/ WealthofNationsconferenceFINAL.pdf. Retrieved on January 20th, 2009.

JOSÉ MANUEL MANEIRO JURJO es Licenciado en Ciencias Económicas y Empresariales por la Universidad de Santiago de Compostela (1992). Doctor en Ciencias Económicas por la Universidad de Alcalá (2004). Profesor de la Universidad de Alcalá. Áreas de Investigación: Economía del Lenguaje, Economía del Patrimonio, Economía Ambiental, Economía Internacional y Desarrollo Económico

DANIEL SOLTECK es Licenciado en Economía y Doctor en Ciencias Económicas. Profesor Titular del Departamento de Fundamentos del Análisis Económico e Historia Económica de la Universidad de Alcalá. Director del Instituto de Estudios Latinoamericanos de la Universidad de Alcalá. Director del Master Internacional de Gestión Universitaria. Áreas de Investigación: Políticas Públicas, Economía Ambiental y Economía del Desarrollo.

RECIBIDO: $24 / 02 / 08$

ACEPTADO: $26 / 11 / 08$

Publicado on-line: 15/06/09

RIS, VOL. 67, No 3, SEPTIEMBRE-DICIEMBRE, 589-607, 2009. ISSN: 0034-9712 